\title{
40 Jahre Kinderschutz im Familienrecht und in der Sozialarbeit
}

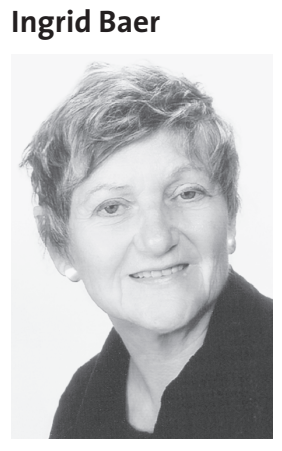

Mitglied der Kommission Zivil-, Familienund Erbrecht, Recht anderer Lebensgemeinschaften im djb, Direktorin des ISD i.R. Oberursel
Kinderschutz ist heute in aller Munde. Er bezieht sich aber nicht nur auf den unmittelbaren Schutz von Kindern vor Gewalt oder Vernachlässigung. Er erfasst ein breites Spektrum von Regelungen insbesondere im Familienrecht, die dafür sorgen sollen, dass Kinder in Situationen, die Risiken für sie enthalten, geschützt werden.

Die Vorstellungen, was für ein Kind richtig und notwendig ist, unterliegen aber durchaus dem Zeitgeist, und das Familienrecht ist, wie wir wissen, ein Spiegel gesellschaftlicher Strukturen. Deshalb lohnt ein Blick zurück.

\section{Nichtehelich geborene Kinder}

Schauen wir uns die rechtlichen Regelungen für das nichtehelich geborene Kind an:

Bis 1971 galt es mit dem Vater als nicht verwandt, obwohl er Kindesunterhalt zahlen musste. Die Mutter hatte nicht die damals so genannte elterliche Gewalt, sondern das Jugendamt wurde automatisch Vormund.

Man war der Meinung, dass der Vater sich sowieso nicht für das Kind interessieren würde, und es auch in seiner Familie keinen Platz finden würde. Die Mutter hielt man wegen ihres „Lebenswandels“ nicht für in der Lage, die alleinige Verantwortung für das Kind zu übernehmen.

Mit der Nichtehelichenrechtsreform von 1971 erhielt die Mutter dann die elterliche Gewalt. Für bestimmte Aufgaben (Unterhaltsansprüche des Kindes und Feststellung der Vaterschaft) gab es jedoch einen Amtspfleger. Dieser entfiel erst 1998, zugunsten der Möglichkeit der Mutter, auf ihren Wunsch eine Beistandschaft des Jugendamtes für diese Aufgaben zu erhalten.

Wurde das Kind von der Mutter zur Adoption freigegeben, so musste auch nach 1971 der Vater nicht zustimmen, er sollte nur gehört werden. Erst 1977 räumte man ihm ein Vorrecht ein, die Adoption selbst zu beantragen. Man hielt es aber im Interesse des Kindes für notwendig, seine Eignung genauso zu prüfen wie bei einer dritten Person.

Ein durchsetzbares Umgangsrecht erhielt der Vater erst mit der großen Reform des Kindschaftsrechts, die 1998 in Kraft trat. Dieses Umgangsrecht war sehr umstritten, weil man fürchtete, dass es zu Konflikten zwischen den Eltern kommen werde, die dem Kindeswohl abträglich sein würden. Die Kinderrechtskonvention der Vereinten Nationen von 1989, die 1992 von der BRD ratifiziert wurde, förderte die Entwicklung. Sie hatte bereits bestimmt, dass jedes Kind das Recht haben solle, mit beiden Elternteilen regelmäßige persönliche Beziehungen zu pflegen. 1998 übernahm das BGB dann diese Regelung und verpflichtete damit die Eltern ihrerseits auch zum Umgang. Inzwischen hatte sich die Erkenntnis durchgesetzt, dass der Umgang mit dem nicht mit dem Kind zusammenlebenden Elternteil jedenfalls in der Regel - für die Entwicklung des Kindes wichtig ist.

Auch die Möglichkeit eines gemeinsamen Sorgerechts des Vaters zusammen mit der Mutter entstand im deutschen Recht erst zu diesem Zeitpunkt. Man hielt es aber im Interesse des Kindes für erforderlich, dass die Mutter damit einverstanden sei und dieses wünschte (gemeinsame Sorgeerklärung), was auch vom Bundesverfassungsgericht 2003 noch einmal als rechtens bestätigt wurde, da das Wohl des Kindes dieses Einvernehmen erfordere. Für den Fall, dass die Mutter ausfällt (z.B. Entzug des Sorgerechts), bestimmte man ebenfalls, dass der Vater das Sorgerecht erhalten solle, allerdings unter der Voraussetzung, dass dies dem Wohl des Kindes dient. Hintergrundüberlegung war hierbei, dass das Kind wichtige Beziehungen zu festen Bezugspersonen entwickelt haben könnte, die ihm wesentlich näher stehen als der abwesende Vater. Hier - wie so oft im Familienrecht - kommt es darauf an, dass von Seiten der Sozialarbeit sorgfältige Abklärungen über die Lebenssituation des Kindes erfolgen, womit die richtige Entscheidung erst möglich gemacht wird. Deshalb ist eine enge Zusammenarbeit zwischen Jugendamt und Familiengericht erwünscht und die Fälle, in denen das Jugendamt vom Vormundschafts- später Familiengericht zu hören ist und auch Stellung zu nehmen hat, wurden sukzessive erweitert.

Weiterhin ist es bereits seit der Sorgerechtsreform von 1980 erforderlich, dass in allen Angelegenheiten, die die Person des Kindes betreffen, dieses nach Möglichkeit von der Richterin oder vom Richter persönlich angehört werden muss. Diese neue Aufgabe war von den Richterinnen und Richtern nicht immer leicht zu lösen, für die Kindesanhörung wurden besondere Richterfortbildungen angeboten.

Anlässlich dieser Reform wurde auch der Begriff „elterliche Gewalt“ abgeschafft und durch „elterliche Sorge" ersetzt. Hintergrund war, dass man das Kind zunehmend als eigenständige Rechtspersönlichkeit ansah, die nicht einem Gewaltverhältnis unterliegen, sondern von den Eltern Schutz und Fürsorge erhalten sollte. 


\section{Scheidung}

Ein weiterer konfliktträchtiger Bereich ist die Scheidungssituation, wenn Paare mit gemeinsamen Kindern betroffen sind.

Hier sah man stets eine Problematik für das Kind enthalten, die nach früherem Recht immer eine Entscheidung des - damals Vormundschaftsgerichts - über die elterliche Gewalt erforderlich machte. Bis zur Reform des Scheidungsrechts 1972 spielte hierbei eine wichtige Rolle, ob ein Elternteil an der Scheidung für allein schuldig erklärt wurde. Zwar ging es auch damals schon um das Kindeswohl, aber man glaubte, dass der Elternteil, der die Ehe „zerstört“ hatte, auch für das gemeinsame Kind weniger Interesse aufbringen würde. Als der Schuldvorwurf bei der Scheidung abgeschafft und die Zerrüttung der Ehe als maßgeblich angesehen wurde, entfiel dieses Argument. Man bezog sich nun nur noch auf das Kindeswohl, wobei man versuchte festzustellen, zu welchem Elternteil das Kind die engeren Bindungen hatte. Man führte das Verbundverfahren ein, bei dem möglichst gleichzeitig über die Scheidung und über das Sorgerecht entschieden werden sollte. Letzteres zeigte sich dann als in der Praxis nicht günstig, weil sich oft erst zu einem etwas späteren Zeitpunkt abzeichnete, wo das Kind leben würde. Die Sorgerechtsentscheidung wurde dem neu gebildeten Familiengericht zugewiesen, ein Fortschritt im Interesse des Kindes. Es blieb jedoch dabei, dass das elterliche Sorgerecht nur einem Elternteil allein gerichtlich zugewiesen wurde, bis 1982 das Bundesverfassungsgericht dies für verfassungswidrig erklärte. Man hatte geglaubt, im Interesse des Kindes Klarheit schaffen zu müssen, zu welchem Elternteil es gehörte und wer andererseits nur Besuchselternteil sein sollte. Das Bundesverfassungsgericht stellte fest, dass es vom Kindeswohl her nicht gerechtfertigt sei, einen Elternteil immer von der elterlichen Sorge auszuschließen, selbst dann, wenn die Eltern erklärten, auch nach der Scheidung gemeinsam weiter für das Kind sorgen zu wollen. Daraus ergaben sich allmählich mehr Entscheidungen, bei denen man es den Eltern zutraute, dass sie gemeinsam das Sorgerecht weiter ausüben würden. Den Hintergrund bildete eine neuere Entwicklung in der Sozialarbeit, nämlich die sogenannte systemische Sicht. Man erkannte, dass das Kind in mehreren wichtigen Bezugssystemen lebt, insbesondere in der erweiterten Familie, dem Freundeskreis etc. Es entwickelte sich eine Väterforschung, die leicht belegen konnte, dass - wenn der geschiedene Vater weiter den Kontakt zu dem Kind hielt - die Beziehung zu ihm für das Kind sehr wichtig blieb. Es kam auch öfters vor, dass ein Kind im Laufe seiner weiteren Entwicklung seinen Lebensmittelpunkt zum anderen Elternteil verlegen wollte, wenn ihm dies möglich gemacht wurde. Es reifte allmählich die Erkenntnis heran, dass man dem Kindeswohl wesentlich besser gerecht werden konnte, wenn es den Eltern gelang, sich auch nach ihrer Trennung und Scheidung in Bezug auf das Kind gegenseitig abzustimmen und zu versuchen, dem Kind nach Möglichkeit beide Eltern in gemeinsamer Sorge zu erhalten.

1998 wurde Abstand genommen von dem Prinzip der zwangsweisen Staatsintervention zur Regelung des Sorgerechts im Scheidungsfall. Stattdessen beließ man es beim gemeinsamen Sorgerecht, das nur auf Antrag eines Elternteils und nach Prü- fung des Kindeswohls auf einen Elternteil allein übertragen werden konnte. Die Jugendhilfe hatte die Aufgabe, die Eltern bei auftretenden Konflikten zu unterstützen und ihnen, ebenso wie dem Kind, Beratung zur Verfügung zu stellen (\$17 SGB III).

Es scheint so, dass dieses neue System sich bewährt hat, zunächst natürlich, indem die Scheidung weniger Konfliktpotential enthält, insbesondere aber in dem Sinne, dass die nicht mit dem Kind zusammenlebenden Elternteile den Kontakt zum Kind besser halten. Freilich gibt es kein Patentrezept, um zu verhindern, dass manche Eltern weiterhin ihren Paarkonflikt zu Lasten des Kindes austragen, nunmehr dann über Umgangsstreitigkeiten.

\section{Kinderschutz}

Ein weiterer Bereich, nunmehr des unmittelbaren Kinderschutzes, hat ebenfalls einschneidende Änderungen erfahren, und zwar der Schutz des Kindes vor den eigenen Eltern. Es kann sich hierbei um Vernachlässigung, Misshandlung oder Missbrauch handeln. Das Recht und die Pflicht der Eltern, ihr Kind selbst nach eigenen Vorstellungen zu erziehen, wird garantiert durch Art. 6 des Grundgesetzes und ist ein wichtiges Merkmal eines demokratischen Rechtssystems. Die Grenzen dieses Elternrechts finden sich aber dort, wo das Kind gefährdet wird, und dann ist zu seinem Schutz das staatliche Wächteramt aufgerufen, also Polizei, Jugendamt, Familiengericht. Aber wo ist diese Grenze zu ziehen?

Verständlicherweise war die Formulierung des zu diesem Zweck geschaffenen $\$ 1666$ BGB zunächst so ausgerichtet, dass man den Eingriff ins Elternrecht nur vornahm, wenn den Eltern ein Schuldvorwurf zu machen war. Die Praxis zeigte aber, dass es sehr wohl Fälle gab, in denen man den Eltern einen Schuldvorwurf nicht machen konnte, beispielsweise, weil sie einfach nicht fähig waren, ein Kind ordnungsgemäß zu versorgen, was die verschiedensten Ursachen haben konnte.

Deshalb korrigierte man 1980 den $\$ 1666$ BGB in der Form, dass auch unverschuldetes Versagen oder eine Gefährdung, die durch das Verhalten eines Dritten hervorgerufen wurde, den Eingriff ins Elternrecht möglich machten, etwa durch Fremdunterbringung des Kindes auch gegen den elterlichen Willen.

Gleichzeitig erkannte man aber die Notwendigkeit, die Eltern in ihrer Erziehungsfähigkeit zu unterstützen und ihnen ambulante Hilfen anzubieten ( $\$ 1666$ a BGB). Das Jugendamt versuchte, von seinem schlechten Ruf als „Kinderwegnehmbehörde“ wegzukommen und sich zu einer beratenden und unterstützenden staatlichen Einrichtung zu entwickeln. Man schuf den Erziehungsbeistand und die Familienhelferin, man baute Kindergärten aus und schuf Hortplätze, um Familien zu entlasten. Auch die Möglichkeiten der Tagespflege in einer fremden Familie für Kinder unter drei Jahren wurden gefördert. Die Diskussion um den Ausbau von Krippenplätzen, nicht nur zur Hilfe für überforderte oder beidseits berufstätige Eltern, sondern auch zur Verbesserung der frühkindlichen Bildung ist auch heute wieder sehr aktuell.

Gravierende Fälle von Kindern, die durch elterliche Vernachlässigung oder Misshandlung zu Tode kamen, ereigneten sich trotz aller fortschrittlichen Bemühungen vermehrt in den letzten Jahren. Man erkannte, dass es nicht genügte einzugreifen, wenn 
das Kindeswohl deutlich gefährdet war. Es erwies sich als notwendig, auch dann schon tätig werden zu können, wenn gewichtige Anhaltspunkte für die Gefährdung des Kindeswohls erkennbar werden, und man fügte 2005 eine entsprechende Regelung in SGB VIII ( $\left.\int 8 \mathrm{a}\right)$ ein. Normiert wurde hierbei wiederum für die Jugendhilfe die Notwendigkeit des Gesprächs mit den Erziehungsberechtigten und dem Kind selbst - soweit die Situation dies zuließ -, mit dem Ziel, einvernehmliche Lösungen zu erreichen.

In jüngster Zeit ging man noch einen Schritt weiter: das Tatbestandsmerkmal des elterlichen Erziehungsversagens hatte sich noch immer als zu hohe Hürde erwiesen, so dass die Familiengerichte von Seiten der Jugendhilfe zu spät eingeschaltet wurden. Es wurde eine neue gesetzliche Regelung getroffen, die am 1. Juli 2008 in Kraft trat. $\$ 1666$ BGB wurde erneut geändert, indem nunmehr die Gefährdung des Kindeswohls oder des Kindesvermögens sowie der mangelnde Wille oder die mangelnde Möglichkeit der Eltern dem abzuhelfen, genügen, damit das Familiengericht Maßnahmen ergreifen kann. Zugleich hat man aber in Abs. 3 eine Reihe von niederschwelligen Maßnahmen aufgezählt, die die Gefährdung beenden sollen, ohne das Elternrecht wesentlich einzuschränken. Das Familiengericht soll damit mehr Möglichkeiten erhalten, tätig zu werden, was auch die Jugendhilfe unterstützt, weil die Eltern dadurch mehr unter Druck stehen, angebotene Hilfen auch anzunehmen. Auch hier wird dem Gespräch mit den Erziehungsberechtigten breiter Raum gegeben, um deren Mitarbeit zu gewährleisten (das sogenannte Erörterungsgespräch). Ein Vorrang- und Beschleunigungsgebot im Verfahren für diese Fälle ergänzt die Regelung. Sieht das Gericht keine Notwendigkeit, Maßnahmen zu ergreifen, so soll es seine Entscheidung nach etwa drei Monaten nochmals überprüfen.

\section{Verfahrenspflegschaft}

Einen wichtigen Schritt, um das Wohl des Kindes zu schützen und um seine Position im Gerichtsverfahren zu stärken, ging man bei der Reform von 1998 durch die Einführung der Verfahrenspflegschaft in $\$ 50$ FGG.

Die Verfassungspflegerin oder der Verfahrenspfleger wird vom Gericht bestellt und hat die Aufgabe, das Interesse des Kindes zu vertreten, insbesondere wenn dieses zu dem seiner gesetzlichen Vertreter in erheblichem Gegensatz steht oder wenn Gegenstand des Verfahrens Maßnahmen wegen Gefährdung des Kindswohls sind. Die Praxis in der Zwischenzeit hat gezeigt, dass die Verfahrenspflegschaft von Gerichten wie von Jugendämtern als sehr hilfreich empfunden wird. Die Verfassungspflegerin oder der Verfahrenspfleger hat mehr Zeit, sich intensiv um das Kind und seine Probleme zu kümmern, persönliche Gespräche mit der Familie zu führen und demzufolge konstruktive Vorschläge zur Lösung des Konfliktes zu machen. Leider sieht es derzeit so aus, als werde das Institut der Verfahrenspflegschaft erheblich in seiner Wirksamkeit beeinträchtigt, nachdem in $\mathrm{Zu}-$ kunft die Vergütung mit einer Pauschale abgegolten wird (das neue FamG tritt am 1. September 2009 in Kraft). Dies wird zwangsläufig dazu führen, dass die Zeit, die die Verfahrenspfleger für den einzelnen Fall aufwenden können, bei schwierigen Fällen nicht mehr ausreichen wird.

\section{Adoption}

Abschließend möchte ich noch ein letztes Thema benennen, das besonders interessant ist in seiner Entwicklung: die Adoption.

Die Adoption hat eine lange Tradition, und sie diente ursprünglich dazu, kinderlosen Ehepaaren zu einem Erben zu verhelfen. Sie war als notarieller Vertragsschluss zwischen den Annehmenden und dem zu Adoptierenden ausgestaltet. Soweit der Anzunehmende noch minderjährig war, konnte man sich nicht vorstellen, dass die Adoptiveltern das Kind behalten würden, falls sie noch ein leibliches Kind bekommen sollten. Deshalb mussten die Annehmenden kinderlos und mindestens 50 Jahre alt sein.

In der Zeit nach dem 2. Weltkrieg gab es viele Kriegswaisen, deswegen änderte man diese Altersvorgabe, allerdings erst 1961. Auch mussten die Kinder meist ins Ausland vermittelt werden, insbesondere in die USA, weil es in Deutschland kaum aufnahmefähige Familien gab. Zum Schutz des Kindes entwickelte man - nachdem die erste Notsituation gemeistert war - Kriterien zur Überprüfung der Adoptionsbewerber, die sogenannte Homestudy, und gab die Kinder zunächst nur in Adoptionspflege. Man arbeitete mit Hilfe des Internationalen Sozialdienstes mit ausländischen Adoptionsvermittlungsstellen zusammen, um nachgehende Berichte über das Wohlergehen des Kindes zu erhalten, danach erst wurde die Adoption im Ausland abgeschlossen.

In der Folge entwickelte sich die Adoption zu einer ganz wichtigen Fürsorgemaßnahme auch innerhalb Deutschlands, insbesondere für ungewollt geborene Kinder. Die nichteheliche Geburt war gesellschaftlich schlecht akzeptiert, für die betroffenen Kinder war die Adoptionsfreigabe oft die bessere Lösung. Nun begann man die familiäre Situation von Kindern, die seit längerer Zeit in einem Heim lebten, zu überprüfen und stellte fest, dass viele überhaupt keinen Kontakt mehr zu Angehörigen hatten und dass es eventuell möglich wäre, eine Adoptivfamilie für sie zu finden. Es gab eine starke Bewegung in der Jugendhilfe: Holt die Kinder aus den Heimen! 1977 wurde die Annahme als Kind neu geregelt, sie erfolgte nun nach Prüfung des Kindeswohles durch gerichtlichen Beschluss. Inbesondere wurd ein Adoptionsvermittlungsgesetz neu geschaffen. Zentrale Adoptionsstellen bei den Landesjugendämtern hatten sich überregional um geeignete Annehmende zu bemühen, auch um Kinder, die behindert waren oder besondere psychische Probleme hatten. Adoptiveltern bekamen Beratung und Hilfe, was oft sehr notwendig war. Eltern, die sich weigerten, ihr Kind zur Adoption freizugeben, aber nicht in der Lage oder bereit waren, ihr Kind selbst zu erziehen, wurden zur Adoptionsfreigabe aufgefordert, im Notfall wurde ihre Einwilligung zur Adoption ersetzt. Psychologische Forschungsergebnisse, dass Kinder zu ihrer gesunden Entwicklung feste Bezugspersonen benötigen und am besten in einem familiären Umfeld aufwachsen, waren der Hintergrund dieser Entwicklung.

Die Situation änderte sich, als es in Deutschland zunehmend weniger Kinder gab, die zur Adoption in Frage kamen. Die verbesserten Möglichkeiten der Geburtenkontrolle und die Akzeptanz auch der nichtehelichen Geburt waren die Ursachen. Es begann in den 1980er Jahren die Adoption von Kindern aus Entwicklungsländern, die dort ohne Familie im Kinderheim leb- 
ten, entweder weil sie Waisen waren oder außerhalb einer Ehe geboren oder auch Mischlingskinder.

Es bildeten sich Bewegungen von kinderlosen Paaren, die sich um Adoption eines ausländischen Kindes bemühten, und es wurden entsprechende internationale Verfahren entwickelt. Es gab aber auch Gegenstimmen, die davor warnten, Kinder zu entwurzeln. Übereinkommen und sozialarbeiterische Schutzvorschriften entwickelten sich auf internationaler Ebene, sowie restriktive gesetzliche Regelungen in den betreffenden Herkunftsländern, um der Inlandsadoption den Vorzug zu geben, soweit es hierfür Möglichkeiten gab. Diese Entwicklung hält bis heute an.

Eine wichtige Erkenntnis war es, den Kindern so viel wie möglich an Informationen über ihre Herkunft zu erhalten, damit sie später ihre Wurzeln besser verstehen könnten. Während man früher die Adoption so anonym wie möglich behandelte, um zu verhindern, dass leibliche Eltern die Geborgenheit des Kindes in der Adoptivfamilie stören könnten, bemüht man sich heute sogar um sogenannte offene Adoptionen, bei denen ein gewisser Kontakt zwischen leiblicher und Adoptivfamilie besteht, sofern die Beteiligten hierzu bereit sind.

Es ist zu beobachten, dass in den letzten Jahren der Adoption gegenüber eine eher kritische Haltung eingenommen wird, sehr zu Unrecht, wie ich meine. Gerade die schlimmen Fälle von hoffnungslos überforderten Eltern, die ihr Kind schwer misshandeln oder vernachlässigen, zeigen, dass sich hier die Jugendhilfe rechtzeitig um Adoptionsberatung hätte bemühen sollen, letztlich auch im Interesse dieser Eltern.

Die Adoption als ganz wichtige und sehr effektive Schutzmaßnahme für gefährdete Kinder bleibt in ihrer Bedeutung bestehen.

\section{Flugblatt zum schuldrechtlichen Versorgungsausgleich}

Mit diesem „Flugblatt“ richten wir uns vor allem an die Anwältinnen unter unseren Mitgliedern: Sie können es als Vorlage für ein Mandantenrundschreiben nutzen oder in der Kanzlei auslegen, um Mandantinnen auf das Problem aufmerksam zu machen. Es wurde vom djb auch als Presseerklärung herausgegeben.

\section{Frauen, küsst Eure Rente wach! Rentenansprüche für geschiedene Frauen im „Dornröschenschlaf“:}

Der Deutsche Juristinnenbund (djb) appelliert an geschiedene Frauen im Rentenalter, ihre Rentenansprüche überprüfen zu lassen und empfiehlt, eine Fachanwältin für Familienrecht oder eine Rentenberaterin aufzusuchen.

Im Rahmen der Scheidung werden oft nur die gesetzlichen Rentenanwartschaften ausgeglichen und ein Restanspruch bleibt dem sogenannten schuldrechtlichen Versorgungsausgleich vorbehalten. Das kommt vor allem in Betracht, wenn der ausgleichspflichtige Ehepartner Betriebsrentenanwartschaften hatte, die nicht oder nur teilweise in ausgeglichen wurden, etwa weil der Höchstbetrag einer gesetzlichen Rente überschritten oder ein betriebliches Versorgungsanrecht verfallbar war.

In all diesen Fällen bleibt der schuldrechtliche Versorgungsausgleich vorbehalten, d.h. er wird später und nur direkt zwischen den geschiedenen Ehepartnern durchgeführt.

Das Problem: Die Durchführung geschieht nicht automatisch, sondern nur auf Antrag der Berechtigten. Der djb weist darauf hin, dass das bei geschiedenen Frauen oft in Vergessenheit gerät, insbesondere, weil zwischen Scheidung und Renteneintritt oftmals viele Jahre oder sogar Jahrzehnte liegen. Im Scheidungsurteil findet sich ein Hin- weis auf den schuldrechtlichen Versorgungsausgleich oft nur in den Gründen; im Urteilsausspruch muss das Gericht das nicht erwähnen. Die Regelung zum Versorgungsausgleich ist ohnehin schon kompliziert und die Berechtigten wissen oft nicht, dass sie diese Rentenrechte noch einmal geltend machen müssen. Und nicht jede Frau heftet ihre Scheidungsunterlagen nach der Scheidung gleich in ihrem Rentenversicherungsordner ab, was unbedingt zu empfehlen ist, damit sämtliche Ansprüche vollumfänglich und rechtzeitig geltend gemacht werden.

Wie die Rechtsanwältinnen und Richterinnen des djb aus der Praxis wissen, werden Ansprüche aus dem schuldrechtlichen Versorgungsausgleich nur selten geltend gemacht. Rentenansprüche erheblichen Ausmaßes werden nur von ganz wenigen Berechtigten abgerufen und liegen bei den Versorgungsträgern „auf Abruf“ bzw. kommen noch dem „Ex“ zugute.

Der schuldrechtliche Versorgungsausgleich kann beim Familiengericht beantragt werden. Voraussetzung ist, dass der ausgleichspflichtige Ehepartner mittlerweile Rente bezieht und der berechtigte Ehepartner entweder ebenfalls Rente bezieht oder das 65. Lebensjahr vollendet hat. Der Anspruch berechnet sich nach der Höhe der Rente, die bisher nicht ausgeglichen wurde.

Möglicherweise kommt sogar ein Ausgleich in Betracht, wenn der ausgleichspflichtige Ehepartner schon gestorben ist. Der Träger einer in Deutschland bestehenden Versorgung, der bei Fortsetzung der Ehe eine Witwenrente gezahlt hätte, muss nach dem Tod des verpflichteten Ehepartners die Zahlung der schuldrechtlichen Ausgleichsrente übernehmen. Die Hinterbliebenenrente eines weiteren Ehepartners wird entsprechend gekürzt. 